O. Huxhold

S. Schäfer

U. Lindenberger

\section{Wechselwirkungen zwischen Sensomotorik und Kognition im Alter}

\author{
Überblick über ein internationales Forschungsfeld
}

Eingegangen: 12. Juni 2008

Akzeptiert: 3. Juli 2008

Online publiziert: 20. Oktober 2008

Oliver Huxhold ( $\bullet)$

Deutsches Zentrum für Altersfragen

Manfred-von-Richthofen-Straße 2

12101 Berlin, Germany

E-Mail: oliver.huxhold@dza.de

Sabine Schäfer

Max-Planck-Institut für Bildungsforschung

Lentzeallee 94

14195 Berlin, Germany

E-Mail: schaefer@mpib-berlin.mpg.de

Ulman Lindenberger

Max-Planck-Institut für Bildungsforschung

Lentzeallee 94

14195 Berlin, Germany

E-Mail:

seklindenberger@mpib-berlin.mpg.de

\section{Age-associated interactions of sensorimotor and cognitive functions}

Zusammenfassung In der letzten Zeit hat die Untersuchung des Verhältnisses zwischen Körper und Geist im Alter in der internationalen Forschung erheblich an Bedeutung gewonnen. Übereinstimmend weisen die meisten Studien darauf hin, dass der $\mathrm{Zu}-$ sammenhang zwischen Sensomotorik und Kognition im Laufe des Erwachsenenalters zunimmt. Die Ursachen hierfür sind komplex. Diese Überblicksarbeit integriert die Ergebnisse korrelativer, experimenteller und neurophysiologischer Ansätze in eine umfassende Darstellung des aktuellen Forschungsstandes. Dabei wird deutlich, dass die zunehmende Interdependenz der beiden Funktionsbereiche die Risiken und Chancen für die Aufrechterhaltung eines mobilen und unabhängigen Lebens im Alter wesentlich beeinflusst.

\section{Schlüsselwörter Sensorik -} Sensomotorik - Kognition Normales Altern

Abstract Research on crossdomain couplings between sensorimotor and cognitive functions in older adults has gained momentum during recent years. Results of most studies point to increasing interdependencies between the two functional domains with advancing adult age. The causes of this increase are complex and show dynamic interactions across adult development. This review integrates experimental, correlational, and neurophysiological evidence, with an eye on implications for maintaining an independent and mobile lifestyle in old age.

Key words sensorimotor cognitive function normal aging
Die Funktionsfähigkeit der Sensomotorik und der Kognition bestimmt entscheidend mit, inwieweit ein mobiles und unabhängiges Leben möglich ist. Aus diesem Grund ist die Erforschung der Entwicklung sensomotorischer und kognitiver Prozesse im Alter nicht nur von akademischem Interesse, sondern hat auch eine unmittelbare Beziehung zur gerontologi- schen Praxis, denn der Alterungsprozess ist insbesondere im hohen Erwachsenenalter durch die Abnahme sensomotorischer und kognitiver Fähigkeiten gekennzeichnet $[15,22]$. In der Forschung wurden diese altersbedingten Veränderungen lange Zeit weitgehend unabhängig voneinander betrachtet. Erst in den 90er Jahren erfuhr die Anschauung, dass alters- 
abhängige Funktionsverluste in der Sensomotorik und der Kognition miteinander verknüpft sind, eine verstärkte Aufmerksamkeit. Inzwischen sind eine ganze Reihe von empirischen Arbeiten publiziert worden, die sich dieser Thematik annehmen [25].

\section{Die Entwicklung der Verbindung von Körper und Geist im Alter}

Leistungen im sensomotorischen und kognitiven Bereich korrelieren querschnittlich mit steigendem Alter zunehmend stärker $[3,13,25]$. Baltes und Lindenberger [3] konnten bspw. zeigen, dass die gemeinsame Varianz zwischen Sensorik und Intelligenz von 11\% bei Personen zwischen 25-69 Jahren auf 31\% in der Gruppe der 70- bis 100-Jährigen anstieg. Auch in Längsschnittstudien waren negative Veränderungen über mehrere Jahre in beiden Leistungsbereichen miteinander korreliert [1]. Korrelationsstudien in diesem Kontext untersuchten eine breite Palette sensomotorischer Variablen, die von visuellen, auditiven und taktilen Leistungen über verschiedene Maße der Muskelstärke bis hin zu aeroben Fitnessmaßen reichten. Diese Bandbreite sensomotorischer Funktionen verdeutlicht die Allgemeingültigkeit des altersassoziierten Zuwachses der Beziehung zwischen den beiden Funktionsbereichen.

Korrelative Studien dieser Art können jedoch nicht darüber Aufschluss geben, ob sich Veränderungen in der Sensorik auf Altersverläufe in der Kognition auswirken oder ob die Wirkrichtung umgekehrt verläuft. Aus diesem Grund nutzten Ghisletta und Lindenberger [9] die zeitliche Struktur der Berliner Altersstudie, um mit Hilfe eines so genannten Bivariate dual-change score models [21] zu bestimmen, ob frühere Leistungen in der Sensorik Veränderungen in der Kognition über einen allgemeinen Alterstrend hinaus vorhersagen können und umgekehrt. Die Autoren konnten zeigen, dass sich altersbedingte Veränderungen in Nahsicht und Fernsicht und in den Intelligenzbereichen verbales Wissen und Wahrnehmungsgeschwindigkeit über einen Zeitraum von 6 Jahren über die Alterstrends in den Funktionsbereichen hinaus wechselseitig dynamisch beeinflussten. Die Einflüsse von der Sensorik auf die Intelligenz und die Wirkung der Intelligenz auf die Sensorik waren jedoch nicht unterschiedlich stark. Im Einklang mit der Common-Cause-Hypothese spricht dieser Befund für einen gemeinsamen dritten Faktor, der auf beide Bereiche in ähnlicher Weise wirkt. Die "Common Cause"-Hypothese besagt, dass sich altersbedingte neurophysiologische Veränderungen negativ auf Prozesse auswirken können, die sowohl an der Sensomotorik als auch der Kognition beteiligt sind. Mit stärkerer Belastung dieser Prozesse kommt es zu einer steigenden funktionsübergreifenden Korrelation [17].

\section{Vom Geist zum Körper - Effekte der Kognition auf die Sensomotorik}

Die Permeationshypothese besagt, dass die schwindende Effizienz der Sensomotorik ältere Menschen dazu zwingt, mehr Aufmerksamkeit auf ihre Bewegungen und Sinne zu richten als jüngere Menschen [19]. Daraus lässt sich folgern, dass die Leistungen Älterer insbesondere dann beeinträchtigt sind, wenn sie eine sensomotorische und eine kognitive Aufgabe gleichzeitig verrichten. Wenn sich z. B. ein 85-Jähriger und ein 25-Jähriger unterhalten und eine belebte Straße überqueren, so wird der 85 -jährige vermutlich das Gespräch unterbrechen, um seine Aufmerksamkeit auf die Überquerung der Straße zu richten, während der 25-Jährige eher in der Lage sein wird, das Gespräch aufrechtzuerhalten. Häufig werden Doppelaufgaben-Experimente genutzt, um dieses Problem unter kontrollierten Bedingungen zu erfassen. Hierbei kann untersucht werden, wie sich die Interaktion von Sensomotorik und Kognition bei älteren Menschen in konkreten experimentell kontrollierten Situationen gestaltet, die möglichst alltagsnah gestaltet sein sollten.

Lindenberger und Kollegen [19] sowie $\mathrm{Li}$ und Kollegen [12] betonen, dass eine Untersuchung des Ressourcenkonflikts zwischen sensomotorischen und kognitiven Aufgaben erfordert, dass zuvor bestehende Leistungsunterscheide - insbesondere Altersunterschiede - in der Analyse berücksichtigt werden. Dies kann erreicht werden, indem alle Versuchsteilnehmer zunächst in den Einzelaufgaben auf das gleiche Leistungsniveau gebracht werden oder indem mehrere Aufgaben mit überlappenden Schwierigkeitsgraden verwendet werden. Außerdem sollte sich der Altersvergleich auf die relativen Kosten beziehen, d.h. auf die Funktionsabnahme unter Doppelaufgabenbedingungen in Relation zur individuellen Leistung bei getrennter Bearbeitung der Aufgaben.

Es sind eine ganze Reihe von Studien erschienen, die belegen, dass ältere Menschen bei der simultanen Bearbeitung sensomotorischer und kognitiver Aufgaben im Vergleich zu jüngeren Studienteilnehmern überproportional starke Leistungseinbußen zeigen [13]. Lindenberger et al. [19] konnten zusätzlich zeigen, dass der Leistungsabfall bei gleichzeitigen Anforderungen an Sensomotorik und Kognition nicht erst im Alter auftritt. Sie trainierten drei Gruppen von Erwachsenen im Alter von 20-30 Jahren, 40-50 Jahren und 60-70 Jahren zunächst in einer Gedächt-

\begin{tabular}{l|l} 
& \\
Z Gerontol Geriat 2 & 2009
\end{tabular} 
nisaufgabe, bis sie ein bestimmtes, zuvor definiertes Leistungsniveau erreicht hatten. Weiterhin übten die Versuchspersonen, so schnell und genau wie möglich auf engen Pfaden zu gehen. Wenn beide Aufgaben gleichzeitig ausgeführt wurden, zeigten alle Altersgruppen sowohl in der Laufleistung als auch in der Gedächtnisleistung Doppelaufgabenkosten. Funktionsübergreifende Ressourcenkonflikte stiegen mit dem Alter der Teilnehmer an. Die Verbindung zwischen Sensomotorik und Kognition besteht also während des gesamten Erwachsenenalters, wird aber mit zunehmendem Alter stärker. Doch unter welchen Bedingungen wirkt sich ein Ressourcenkonflikt eher $\mathrm{zu}$ Lasten der sensomotorischen oder eher zu Lasten der kognitiven Leistungen aus?

Li und Kollegen [14] integrierten Hindernisse in eine enge Laufbahn, über die eine Gruppe 20- bis 30-Jähriger und eine Gruppe 60-70 Jahre alter Personen ging. Zudem passten sie unter einigen Bedingungen die Schwierigkeit der gleichzeitig auszuführenden Gedächtnisaufgabe an das Leistungsniveau jedes Probanden an. Unter dieser Bedingung zeigten die älteren Erwachsenen im Verhältnis zu der jüngeren Gruppe einen deutlich stärkeren Leistungsabfall in der Gedächtnisleistung als in der Laufgeschwindigkeit. Offenbar halten Ältere in Situationen, in denen das Gleichgewicht bedroht ist, ihre sensomotorischen Fähigkeiten auf Kosten der Leistungen im kognitiven Bereich aufrecht. Diese Umverteilung von Ressourcen ist nicht nur von der tatsächlichen Bedrohung des Gleichgewichts abhängig, sondern kann auch durch eine wahrgenommene Bedrohung ausgelöst werden. Brown und Kollegen [4] ließen ältere Probanden die gleiche Doppelaufgabe - Balancieren bei gleichzeitiger Bearbeitung einer Gedächtnisaufgabe - einmal am Boden und einmal in 1 Meter Höhe durchführen. In 1 Meter Höhe opferten die Älteren ihre kognitive Leistungsfähigkeit zu Gunsten der Balance, am Boden nicht. Diese Fähigkeit, Ressourcen zwischen Bereichen umzuverteilen, erscheint im Alter besonders wichtig, denn gesundheitliche Konsequenzen eines Sturzes können bei Älteren schwerwiegend sein.

Allerdings ist es nicht immer sinnvoll, kognitive Ressourcen in eine sensomotorische Aufgabe zu investieren. Huxhold und Kollegen [10] konnten zeigen, dass sowohl jüngere als auch ältere Erwachsene ihre Gleichgewichtsleistung im aufrechten Stand verbesserten, wenn sie zusätzlich eine leichte kognitive Aufgabe bearbeiteten. Die Autoren weisen darauf hin, dass milde Formen kognitiver Belastung im Alltag eher die Regel als die Ausnahme sind. Aus diesem Grund sei es besser, keine Aufmerksamkeit auf die Motorik zu richten, solange die individuellen sensomotorischen Fähigkeiten hoch oder die zu bewältigenden Aufgaben einfach sind. Unter diesen Be- dingungen ist die Motorik weitgehend automatisiert. Wird Aufmerksamkeit auf automatisierte Prozesse gerichtet, werden diese gestört und die motorische Leistung verschlechtert sich.

Die Störung kognitiver Aufgaben durch den Aufmerksamkeitsbedarf der Sensomotorik kann sich negativ auf die Mobilität älterer Menschen im Alltag auswirken. Mobilität im Alter wird nicht allein durch eine intakte Motorik, sondern auch durch die Fähigkeit gewährleistet, sich im Raum orientieren zu können. Dass dieser Aspekt besondere Beachtung verdient, demonstriert eine Studie, in der jüngere und ältere Personen auf einem Laufband liefen, während sie sich in einer virtuellen Umgebung zurechtfinden sollten [20]. Hier zeigte sich, dass die Navigationsleistung der älteren Versuchsteilnehmer durch eine einfache Entlastung der Sensomotorik, nämlich der Möglichkeit, sich an einem Geländer festzuhalten, um ca. 25\% verbessert wurde. Auf die Navigationsleistung der Jüngeren hatte diese Entlastung keinen Einfluss.

\section{Vom Körper zum Geist - Effekte körperlicher Fitness auf die Kognition}

Aus biologischer Sicht hängt körperliche Fitness eng mit der Leistungsfähigkeit kognitiver Systeme zusammen [2]. Dem entspricht die Erwartung, dass körperliche Betätigung neben sensomotorischen auch kognitive Leistungen verbessern kann. Für diese Erwartung gibt es mittlerweile eine Reihe empirischer Belege [7]. Schon eine Studie von 1975 [26] fand, dass ältere Sportler bessere kognitive Leistungen zeigten als ihre nicht sporttreibenden Altersgenossen. Im Längsschnitt fanden Rogers und Kollegen [24] bei Personen, die auf die 65 zugingen, dass diejenigen, die körperlich aktiv waren, geringere altersbedingte, kognitive Leistungseinbußen zeigten.

Die spezifische Wirkung körperlicher Fitness auf kognitive Fähigkeiten im Alter wird vor allem in Interventionsstudien untersucht. Im Allgemeinen werden diese Studien mit kognitiv unauffälligen älteren Menschen durchgeführt, die bislang keinen Sport trieben. Diese Personen werden zufällig verschiedenen Interventionsbedingungen zugeordnet, die unterschiedliche körperliche Trainingspläne und Kontrollbedingungen enthalten können. Häufig werden die Probanden mit einer Reihe unterschiedlicher kognitiver Aufgaben vor und nach dem Training getestet, um die Effekte unterschiedlichen Körpertrainings auf verschiedene kognitive Fähigkeiten zu bestimmen.

Kramer und Kollegen [11] ordneten zufällig 124 Personen im Alter zwischen 60 und 70 Jahren, die bis dahin sportlich nicht aktiv waren, entweder einem Ausdauertraining oder einem Training mit 
Kraft- und Dehnungsübungen zu. Vor und nach der Trainingsphase absolvierten beide Gruppen eine Reihe kognitiver Tests. Nach Abschluss der 6-monatigen Trainingsprogramme zeigte sich, dass sich die körperliche Fitness nur bei den Personen in der Ausdauergruppe signifikant verbessert hatte. Außerdem verbesserten sich nur diese Personen in ihren kognitiven Leistungen. Die Trainingsgewinne waren beschränkt auf Aufgaben, die ein hohes Maß exekutiver Kontrolle benötigten, d.h. Prozesse, die die Auswahl und Unterdrückung von Informationen steuern und koordinieren. Auf einfache Reaktionszeitaufgaben hatte das Training keinen Effekt.

Eine differenzierte Bestimmung des aeroben Trainingseffekts lieferte eine Metaanalyse, die die Ergebnisse von 18 zwischen 1966 und 2001 publizierten Ausdauertrainingsstudien zusammenfasst [6]. Die Metaanalyse zeigt, dass Fitness einen robusten aber selektiven Effekt auf die Kognition hat. Die Effekte eines Trainings sind größer, wenn aerobes und anaerobes Training kombiniert werden. Kurze Trainingsprogramme von 2 Monaten wirken ebenso gut wie mittellange, aber ein wenig schlechter als längere Programme mit über 6 Monaten Dauer. Der positive Einfluss eines Ausdauertrainings ist am stärksten bei kognitiven Aufgaben, die ein großes $\mathrm{Maß}$ an exekutiver Kontrolle benötigen. Dieser Befund ist besonders interessant für die Altersforschung, da kognitive Anforderungen, die viel exekutive Kontrolle benötigen, mit besonders starken Leistungseinbußen einhergehen [27].

Neurophysiologische Untersuchungen zeichnen ein ähnliches Bild. Colcombe, Erickson, Raz et al. [5] untersuchten die Dichte des Gehirngewebes bei älteren Erwachsenen in Abhängigkeit von deren körperlicher Fitness. Mit steigendem Alter war die Dichte insbesondere in frontalen, temporalen und parietalen Bereichen geringer. Dieser Alterseffekt war signifikant reduziert, wenn die Teilnehmer eine hohe körperliche Fitness aufwiesen, auch nachdem konfundierende Variablen in die Analysen einbezogen wurden. Die positiven Effekte körperlicher Fitness waren in den Arealen am stärksten, in denen die altersassoziierten Verluste von Gewebedichte am größten waren [23]. Eine weitere Studie zeigte, dass die Aktivierung von Gehirnzentren in älteren Erwachsenen bei der Bearbeitung kognitiver Aufgaben dem Aktivierungsprofil jüngerer Erwachsener ähnlicher war, nachdem erstere ein Ausdauertraining absolviert hatten [8].

\section{Körper und Geist im Alter: Fazit}

Es ist unstrittig, dass mit zunehmendem Alter der Zusammenhang zwischen Sensomotorik und Kognition stärker wird. Die Gründe für dieses Phänomen sind vielfältig und interagieren auf komplexe Weise. Die neurophysiologische Forschung belegt, dass der normale Alterungsprozess mit Veränderungen in einer Vielzahl neurochemischer, anatomischer und funktionaler Systeme und Strukturen einhergeht. Es ist anzunehmen, dass sich diese seneszenten Veränderungen sowohl auf sensomotorische als auch auf kognitive Prozesse störend auswirken. In ihrer Gesamtheit können diese neurophysiologischen Veränderungen als eine gemeinsame Ursache (Common Cause) verstanden werden, die zu einer zunehmenden Korrelation beider Funktionsbereiche beiträgt. Zusätzlich steigt die Interdependenz zwischen Geist und Körper, wie von der kognitiven Permeationshypothese vorhergesagt, durch bereichsübergreifende Ressourcenkonflikte, die aus der altersbedingten Deautomatisierung sensomotorischer Prozesse entstehen. Zwar interagieren sensomotorische und kognitive Prozesse über die gesamte Lebensspanne. Alterungsbedingt schwindende Kapazitäten erhöhen jedoch die Wahrscheinlichkeit und die Risiken von Ressourcenkonflikten. Aus diesen Gründen investieren ältere Erwachsene mehr Aufmerksamkeit in motorische Prozesse als jüngere Erwachsene. Dies reduziert die Gefahr von Unfällen, birgt jedoch die Gefahr funktionaler Leistungseinbußen im kognitiven Bereich, die sich ebenfalls negativ auf die Mobilität im Alter auswirken können. Interventionsstudien im Bereich der körperlichen Fitness haben jedoch gezeigt, dass ein gezieltes Ausdauertraining die Folgen normaler Alterung für sensomotorische und kognitive Funktionen abmildern kann. Körperliche Fitness hat einen positiven Einfluss auf die Kognition im Alter, insbesondere bei Personen mit niedriger körperlicher Fitness. Interessanterweise sind die Trainingseffekte gerade in jenen funktionellen und neurophysiologischen Bereichen am stärksten, die die größten alterungsbedingten Veränderungen aufweisen.

Abschließend möchten wir aus den empirischen Befunden konkrete Empfehlungen ableiten, welche die Aufrechterhaltung eines ausreichenden Funktionsniveaus über einen längeren Zeitraum unterstützen können.

\section{Körper und Geist im Alter - Empfehlungen für die Praxis}

1. Ältere Menschen sollten regelmäßig Ausdauersport betreiben. Mit aerobem Training werden nicht nur sensomotorische Fähigkeiten verbessert, sondern auch die Kognition kann davon profitieren. Ausdauertraining hat zudem den Vorteil, dass es sich positiv auf eine ganze Reihe kognitiver Fähigkeiten auswirkt und insbesondere solche Fähigkeiten

\begin{tabular}{l|l} 
& \\
Z Gerontol Geriat 2 & 2009
\end{tabular} 
verbessert, die für die Bewältigung komplexer Probleme wichtig sind (exekutive Kontrolle).

2. Das Fähigkeitsniveau in der Sensomotorik entscheidet darüber, ob es sinnvoll ist, Aufmerksamkeit auf die eigenen Bewegungen zu richten oder nicht. Solange Bewegungsprozesse automatisch ablaufen können, verschlechtert die Investition kognitiver Ressourcen die Leistung. Bei den Älteren hängt die Ressourcenallokation unter anderem auch von der wahrgenommenen Bedrohung des Gleichgewichts ab. Aus diesem Grund kann es ratsam sein, im Alter an Maßnahmen teilzunehmen, die das Sicherheitsgefühl in Bezug auf die eigenen sensomotorischen Fähigkeiten angemessen erhöhen.

3. Im Altersverlauf können die sensomotorischen Fähigkeiten auf ein Niveau sinken, bei dem selbst einfache motorische Akte wie Gehen ein so star- kes Maß an Aufmerksamkeit erfordern, dass kognitive Prozesse (bspw. räumliche Orientierung), die für die Mobilität im Alter notwendig sind, in Mitleidenschaft gezogen werden. In besonderen Fällen können diese Einschränkungen nur erkannt werden, wenn beide Fähigkeiten gleichzeitig gefordert werden. Es ist deshalb erforderlich, dass die Diagnostik der Mobilität im Alter Doppelaufgabensituationen mit einbezieht, um eventuelle Ressourcenkonflikte $\mathrm{zu}$ erkennen. Sollte ein solcher Fall vorliegen, könnte es sinnvoll sein, die Sensomotorik oder die Kognition durch externe Hilfsmittel zu unterstützen, obwohl beide Funktionsbereiche allein noch ausreichend funktionsfähig sind. Moderne Informationstechnologien können hierbei eine positive Rolle spielen [16, 18].

Interessenkonflikt Es besteht kein Interessenkonflikt.

\section{Literatur}

1. Anstey KJ, Hofer SM, Luszcz MA (2003 a) A latent growth curve analysis of late-life sensory and cognitive function over 8 years: Evidence for specific and common factors underlying change. Psychol Aging 18:714-726

2. Anstey KJ, Smith GA (1999) Interrelationships among biological markers of aging, health, activity, acculturation, and cognitive performance in late adulthood. Psychol Aging 14: 605-618

3. Baltes PB, Lindenberger U (1997) Emergence of a powerful connection between sensory and cognitive functions across the adult life span: A new window to the study of cognitive aging? Psychol Aging 12:12-21

4. Brown LA, Sleik RJ, Polych MA, Gage WH (2002) Is the prioritization of postural control altered in conditions of postural threat in younger and older adults? J Gerontol A-Biol 57A: M785-M792

5. Colcombe S, Erickson KI, Raz N et al (2003) Aerobic fitness reduces brain tissue loss in aging humans. J Gerontol A-Biol 58A:176-180

6. Colcombe S, Kramer AF (2003) Fitness effects on the cognitive function of older adults: A meta-analytic study. Psychol Sci 14:125-130

7. Colcombe S, Kramer AF, McAuley E, Erickson KI, Scalf P (2004) Neurocognitive aging and cardiovascular fitness: Recent findings and future directions. J Mol Neurosci 24:9-14
8. Colcombe SJ, Kramer AF, Erickson $\mathrm{KI}$ et al (2004) Cardiovascular fitness, cortical plasticity, and aging. P Natl A Sci USA 101:3316-3321

9. Ghisletta P, Lindenberger U (2005) Exploring structural dynamics within and between sensory and intellectual functioning in old and very old age: Longitudinal evidence from the Berlin Aging Study. Intelligence 33:555587

10. Huxhold O, Li S-C, Schmiedek F, Lindenberger U (2006) Dual-tasking postural control: Aging and the effects of cognitive demand in conjunction with focus of attention. Brain Res Bull 69:294-305

11. Kramer AF, Hahn S, Cohen NJ, Banich MT, McAuley E, Harrison CR et al (1999) Ageing, fitness and neurocognitive function. Nature 400:418419

12. Li KZH, Krampe RT, Bondar A (2005) An ecological approach to studying aging and dual-task performance. In: Engle RW, Sedek G, Hecker U v, McIntosh DN (eds) Cognitive limitations in aging and psychopathology. Cambridge University Press, Cambridge UK, pp 190-218

13. Li KZH, Lindenberger U (2002) Relations between aging and sensory/sensorimotor and cognitive functions. Neurosci Biobehav Rev 26:777-783

14. Li KZH, Lindenberger U, Freund AM, Baltes PB (2001) Walking while memorizing: Age-related differences in compensatory behavior. Psychol Sci $12: 230-237$
15. Li S-C, Lindenberger U, Hommel B, Aschersleben G, Prinz W, Baltes PB (2004) Transformations in the couplings among intellectual abilities and constituent cognitive processes across the life span. Psychol Sci 15: 155-163

16. Lindenberger U (2007) Technologie im Alter: Chancen aus Sicht der Verhaltenswissenschaften. In: Gruss $P$ (Hrsg) Die Zukunft des Alterns: Die Antwort der Wissenschaft. Beck, München, S 221-239

17. Lindenberger U, Baltes PB (1994) Sensory functioning and intelligence in old age: A strong connection. Psychol Aging 9:339-355

18. Lindenberger $U$, Lövdén $M$, Schellenbach M, Li S-C, Krüger A (2008) Psychological principles of successful aging technologies: A mini-review. Gerontol 54:59-68

19. Lindenberger $U$, Marsiske $M$, Baltes PB (2000) Memorizing while walking: Increase in dual-task costs from young adulthood to old age. Psychol Aging 15:417-436

20. Lövdén $M$, Schellenbach $M$, Grossman-Hutter B, Krüger A, Lindenberger U (2005) Environmental topography and postural control demands shape aging-associated decrements in spatial navigation performance. Psychol Aging 20:683-694 
21. McArdle JJ (2001) A latent difference score approach to longitudinal dynamic structural equation analyses. In: Cudeck R, duToit S, Sörbom D (eds) Structural equation modeling: Present and future. Scientific Software Intl, Lincolnwood, IL, pp 342380

22. Park DC, Polk TA, Mikels JA, Taylor SF, Marshuetz C (2001) Cerebral aging: Integration of brain and behavioral models of cognitive function. Dialogues in Clin Neurosci 3:151-165
23. Raz N, Lindenberger U, Rodrigues KM et al (2005) Regional brain changes in aging healthy adults: General trends, individual differences and modifiers. Cereb Cortex 15:1676-1689

24. Rogers RL, Meyer JS, Mortel KF (1990) After reaching retirement age physical activity sustains cerebral perfusion and cognition. JAGS 38: 123-128
25. Schäfer $\mathrm{S}$, Huxhold $\mathrm{O}$, Lindenberger U (2006) Healthy mind in healthy body? A review of sensorimotor-cognitive interdependencies in old age. Euro Rev Aging Phys Activity 3: 4554

26. Spirduso WW (1975) Reaction and movement time as a function of age and physical activity level. J Gerontol 43:18-23

27. Verhaeghen P, Cerella J (2002) Aging, executive control, and attention: A review of metaanalyses. Neurosci Biobehav Rev 26:849-857 